\title{
ТЕХНОЛОГИЧЕСКИЙ КОМПЛЕКС ДЛЯ ПЕРЕРАБОТКИ ВЕРМИКУЛИТОВЫХ КОНЦЕНТРАТОВ И КОНГЛОМЕРАТОВ
}

\author{
Нижегородов Анатолий Иванович', \\ nastromo_irkutsk@mail.ru \\ Гаврилин Алексей Николаевич², \\ tom-gawral@list.ru \\ Мойзес Борис Борисович², \\ mbb@tpu.ru
}

\author{
Кувшинов Кирилл Александрович², \\ kuvshinov@tpu.ru
' Иркутский национальный исследовательский технический университет, Россия, 664074, г. Иркутск, ул. Лермонтова, 83.
2 Национальный исследовательский Томский политехнический университет, \\ Россия, 634050, г. Томск, пр. Ленина, 30.
}

\begin{abstract}
Актуальность. Вермикулит и материалы на его основе нашли широкое применения во многих сферах жизнедеятельности: промышленность, природообустройтво, обеспечение пожаробезопасности и т. д. В связи с этим актуально решение задач, связанных сусовершенствованием технологических процессов обжига вермикулитовых концентратов и конгломератов, особенно в аспекте получения высококачественного вспученного вермикулита. В основе обеспечения ресурсоэффективности технологических процессов получения вспученного вермикулита лежат электрические модульно-спусковые печи. Поэтому рассмотрение вопросов разработки новых конструкций электрических модульно-спусковые печей и технологических комплексов на их основе актуально.

Цель исследования: разработка технологического комплекса для переработки вермикулита, изучение указанных технологических процессов и оценка их эффективности.

Методы: анализ источников информации в области исследования, синтез конструктивных и технологических решений, параметрическое и функциональное описание, проведение экспериментальных работ.

Результаты. Разработан технологический комплекс для переработки вермикулитовых концентратов и конгломератов с учетом требований экономии электроэнергии, повышения качества вспученного вермикулита и вторичных целевых продуктов. В ходе работы усовершенствована модульно-спусковая печь, позволяющая экономить до 15 \% электроэнергии при той же производительности. Доказано, что предложенная система охлаждения обеспечивает повышение прочности вспученных зерен на 19,1%, увеличение почти до 90 \% выделения вторичных целевых продуктов. Значительно повышается главный показатель качества снижение насыпной плотности основного целевого продукта за счет выведения вторичных продуктов и инертного материала. Сделан прогноз развития работ в аспекте разработки новой электрической печи с подвижной подовой платформой, обладающей удельной энергоемкостью обжига вермикулита около 81,6 мДж/м³.
\end{abstract}

\section{Ключевые слова}

Технологический комплекс, электрическая модульно-спусковая печь, неэлектрический «нулевой» модуль, эксергия, система охлаждения, система отделения, прочность вермикулита, вторичные целевые продукты, инертный материал, температуропроводность.

\section{Введение}

Вспученный вермикулит, благодаря своим уникальным свойствам, нашел применение в различных производственных сферах [1-14], в связи с чем развитию технологий обработки вермикулитовых руд уделяется большое внимание $[11,14]$.

Совершенствование электрических печей для обжига вермикулитовых концентратов и конгломератов и дополнительных систем, способствующих получению высококачественного целевого продукта - вспученного вермикулита, привело к ряду технических решений, примененных в рассматриваемом комплексе. В его основе электрическая модульно-спусковая печь с дополнительным неэлектрическим модулем, работающим за счет эксергии вермикулита и сопутствующего инертно- го материала. Он включает в себя системы охлаждения вспученного вермикулита, отделения инертного материала и выделения мелкофракционного вермикулита, являющегося вторичным целевым продуктом, а также типовые элементы - бункерыосадители, бункеры-накопители, вентиляторы и воздуховоды.

Обжиг вермикулита в режиме термоудара обеспечивает бо́льшее вспучивание разделившихся слоев, появляется больше крупных пор, а работу вспучивания совершают гидратная и межслоевая вода. Но из электрических модулей он выходит недовспученным, чтобы завершение процесса происходило в дополнительном «нулевом» модуле.

Быстрое охлаждение вермикулита повышает прочность его зерен - важнейший показатель его 
качества, особенно в случаях использования материала в качестве легковесного наполнителя для легких строительных и жаростойких бетонов.

Выделение мелкофракционного вермикулита, с одной стороны, снижает насыпную плотность основного целевого продукта, а с другой - он сам является ценным материалом для приготовления жаростойких красок и мастик.

Целью данного исследования стала разработка комплекса для реализации технологических про- цессов переработки вермикулита, их исследование и оценка эффективности.

\section{Работа технологического комплекса}

Технологический комплекс для переработки вермикулитовых концентратов и конгломератов (рис. 1) имеет в своем составе электрическую модульно-спусковую печь, неэлектрический «нулевой» модуль, систему выделения мелкофракционного вермикулита, охладитель вспученного верми-

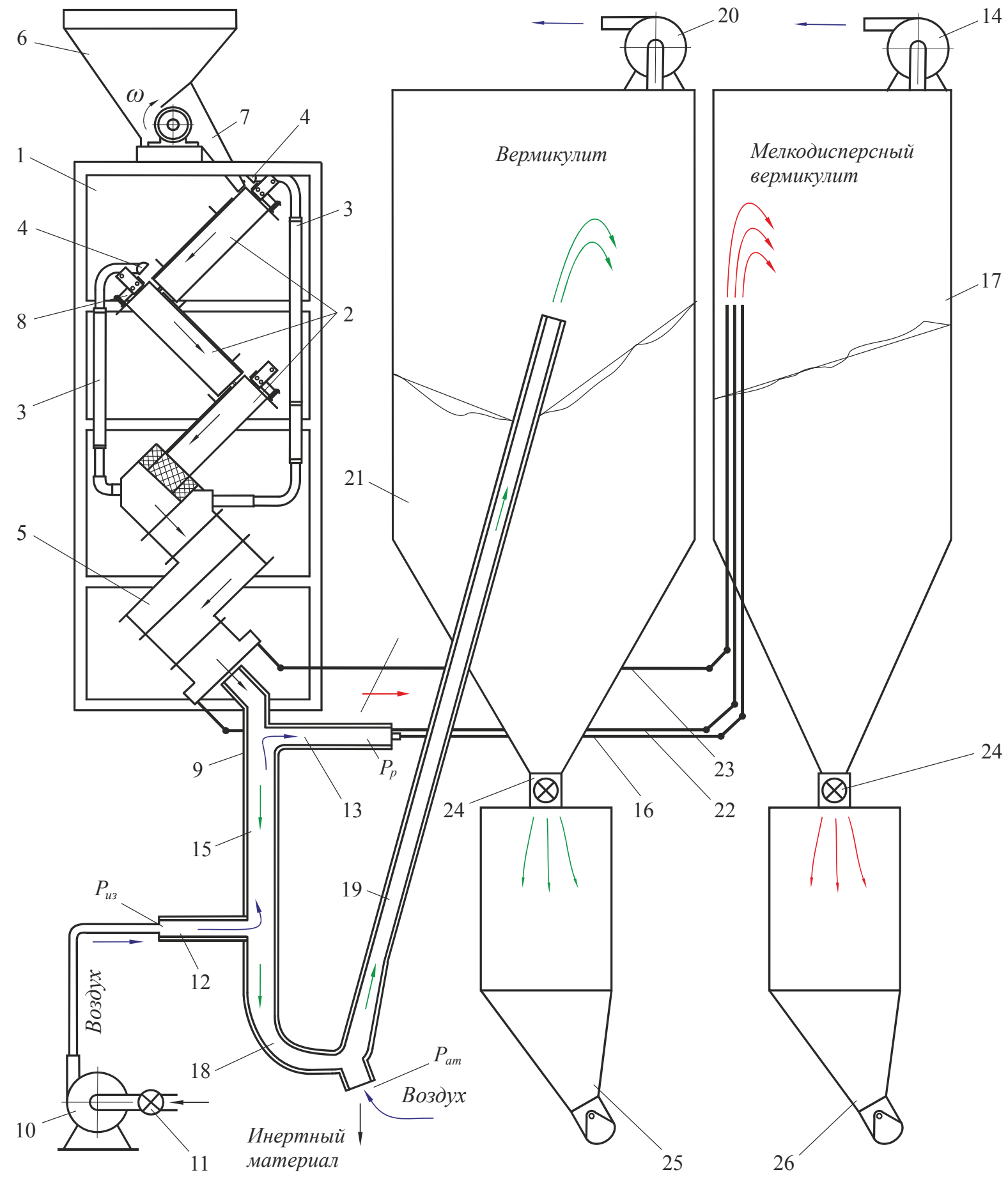

Рис. 1. Технологический комплекс для переработки вермикулитовых концентратов и конгломератов

Fig. 1. Technological complex for processing vermiculite concentrates and conglomerates 
кулита, отделитель инертного материала, бункеры-осадители и бункеры-накопители для целевых продуктов и систему вентиляторов и воздуховодов.

В трехмодульной электрической печи - 1 производится термообработка сырья (модули обжига - 2) и выделение мелкофракционного вермикулита с помощью системы воздуховодов - 3 и трубчатых всасывателей - 4, через которые он всасывается в полости специального «нулевого» модуля -5 . Окончание процессов дегидратации и вспучивания вермикулитовых зерен осуществляется здесь же - в модуле - 5 трубчатого типа, не потребляющего электроэнергию и работающего за счет накопленной в самом вермикулите и инертном материале эксергии.

Вермикулитовый концентрат или конгломерат подается в печь дозатором - 6 по лотку -7 и поступает в модули - 2, состоящие из огнеупорных оснований и термокрышек, образующих замкнутые пространства. На основаниях модулей размещены нагреватели, выполненные их нихромовой ленты и закрепленные на изоляторах - 8. Сырьевой материал пересыпается с одного модуля на другой и, подвергаясь тепловому излучению, дегидратирует и вспучивается, но эти процессы в модулях, длящиеся 2,6-2,8 с, полностью не завершаются. Поток материала проходит через «нулевой» модуль -5 и через $1,5-1,7$ с поступает в охладитель -9 коробчатого сечения.

Вентилятор - 10, снабженный дросселем - 11 во всасывающем патрубке, подает холодный воздух $\left(\sim 20^{\circ} \mathrm{C}\right)$ с небольшим избыточным давлением

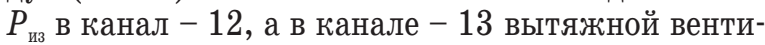
лятор - 14 системы выделения мелкофракционного вермикулита создает разряжение $P_{\mathrm{p}}$.

Перепад давлений в каналах - 13, 12 максимален и бо́льшая часть воздушного потока идет по каналу - 13. При этом в канале - 15 возникает поток воздуха навстречу падающему вермикулиту и инертному материалу. Встречный поток охлаждает вспученные зерна, одновременно отделяет и уносит мелкофракционный вермикулит по трубопроводу - 16 в бункер-осадитель - 17, охлаждая и очищая основной целевой продукт, выпадающий вместе с инертным материалом в сужающийся отвод - 18 продуктопровода - 19. Скорость встречного потока в канале - 15 настраивается дросселем - 11 так, чтобы даже самые мелкие вспученные зерна не могли быть унесены в бункер-осадитель - 17. В этот же бункер, где возникает разряжение от работающего вытяжного вентилято$\mathrm{pa}-14$, поступает вторичный целевой продукт из системы воздуховодов - 3 и трубчатых всасывателей -4 печи - 1 по трубопроводам - 22, 23.

Эффективность охлаждения вермикулита в канале - 15 тем больше, чем больше перепад высот между каналами - 13, 12 и чем больше скорость воздуха: во-первых, из-за более эффективного обдува, а во-вторых, из-за снижения скорости паде- ния вспученных зерен и увеличения времени их пребывания в восходящем потоке в канале - 15 .

В нижней части отвода - 18 расположено отверстие, через которое выпадает тяжелый инертный материал, но из-за работы вытяжного вентилятора - 20 бункера-осадителя вспученного вермикулита - 21 вермикулит всасывается в продуктопровод, сечение которого подобрано так, чтобы для самых крупных частиц скорость витания была меньше средней скорости потока воздуха. При движении вспученных зерен в продуктопроводе они дополнительно охлаждаются.

В канале - 15 создается разряжение, поэтому между корпусом охладителя и «нулевым» модулем выдержан зазор, исключающий подсос горячего воздуха из его рабочего пространства.

Накопившиеся в бункерах-осадителях целевые продукты периодически через секторные затворы 24 , пересыпаются в бункеры-накопители - 25, 26.

На рис. 2 показано устройство «нулевого» модуля. Он содержит патрубки - 1,2 , сообщенные с тепловыми камерами - 5, 6. Внутри корпуса размещен термоизоляционный материал, изолирующий трубчатые рабочие камеры - 7 от тепловых камер - 5, 6, которые, из-за потока горячего воздуха, поступающего от всасывателей - 4 по воздуховодам - 3 (рис. 1) дополнительно снижают отток тепла из камер - 5, 6. Вермикулит из нижнего модуля попадает в полости - 4 и по рабочим камерам, имеющим конические участки, увеличивающие по мере движения его кучность, продвигается к выходу. Здесь происходит завершение дегидратации и вспучивания вермикулита за счет накопленной в нем и инертном материале эксергии.

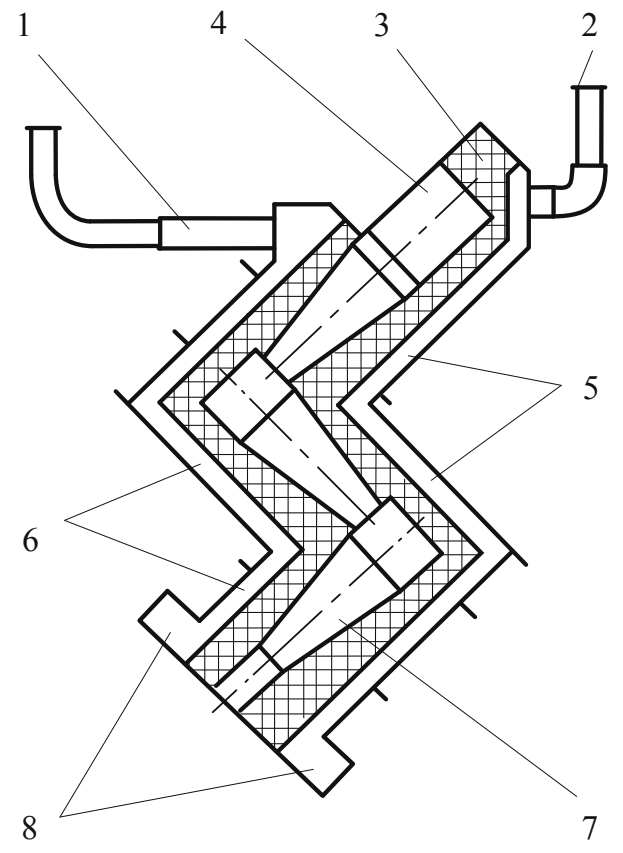

Рис. 2. Дополнительный неэлектрический модуль

Fig. 2. Optional non-electric module 


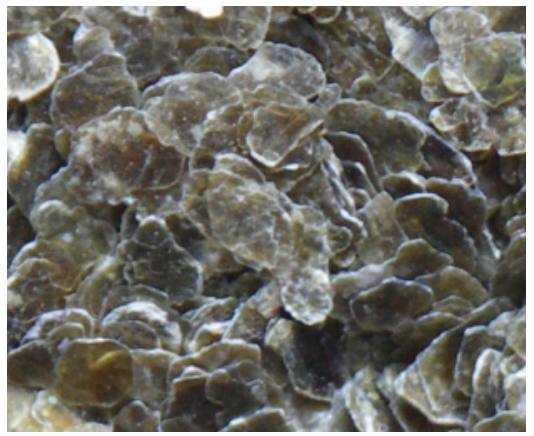

$a / a$

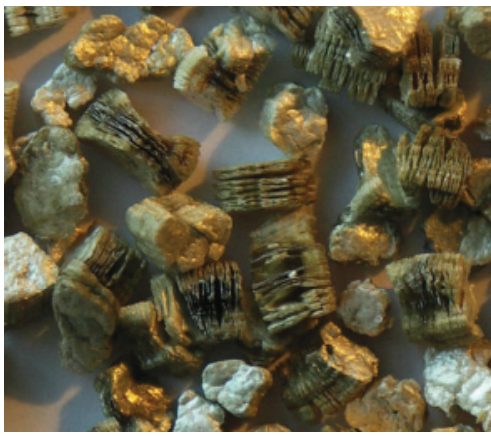

$\sigma / b$

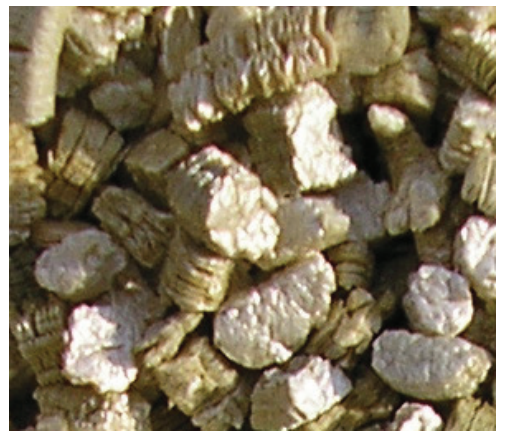

$B / c$

Рис. 3. Стадии вспучивания вермикулита: а) концентрат; б) недовспученность; в) полностью вспученный

Fig. 3. Expansion stages of vermiculite: a) concentrate; b) not completely expanded; c) expanded

\section{Использование эксергии вермикулита и инертного материала}

Эксэргия - это качественная энергия, пригодная к совершению полезной работы. В нашем случае накопленная в вермикулите теплота путем кондуктивного переноса по чешуйкам вглубь зерен способна завершить процессы дегидратации и вспучивания. Ниже показаны стадии структурообразования вермикулита от концентрата (рис. $3, a)$ до полностью вспученного материала (рис. 3, в) с промежуточным состоянием недовспученности (рис. 3, б). Эффект «нулевого» модуля состоит в добавлении времени для завершения указанных процессов. Теплоусвоение вермикулита можно прервать на выходе из нижнего электрического модуля - 2 (рис. 1), например при температуре поверхности зерен $\sim 720{ }^{\circ} \mathrm{C}$, перенести его окончание в «нулевой» модуль, и тем самым снизить потребляемую мощность печи. Оставшаяся теплота в отсутствие внешнего источника энергии будет переходить в глубинные слои зерен.

Но действуют еще два фактора: лучистый и кондуктивный перенос тепла от мелких зерен, в которых процессы уже завершились, на крупные, а также от более плотного инертного материала, нагретого до конечной температуры еще быстрее, на те же крупные зерна (рис. 4).
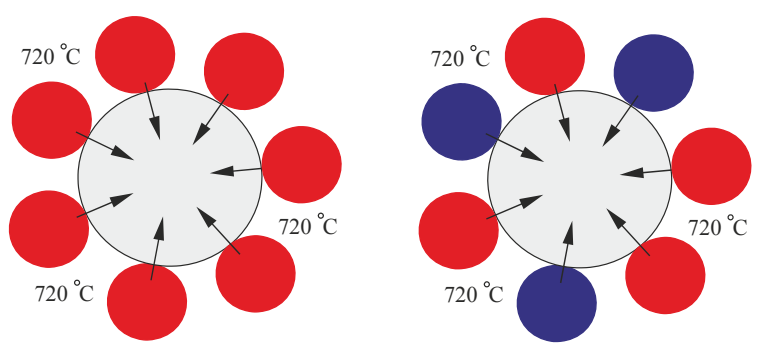

- Инертный материал

Рис. 4. Кластеры, образующиеся в конических трубках дополнительного «нулевого» модуля

Fig. 4. Clusters formed in conical tubes of an optional «zero» module
Электрические модули печи снабжены всасывателями - 4 (рис. 1), поглощающими утечки лучистой энергии и тепло конвективных потоков, идущие через верхние торцы модулей обжига. Поток теплоносителя - нагретый до $380-400{ }^{\circ} \mathrm{C}$ воздух, прокачивается через тепловые камеры $-5,6$ (рис. 2), практически исключая потери накопленной зернами эксергии.

Теоретические модели процессов теплопереноса рассматривались в предшествующих работах авторов, поэтому здесь рассмотрены результаты экспериментов, проведенных на модульно-спусковой печи с дополнительным неэлектрически модулем (рис. 5).

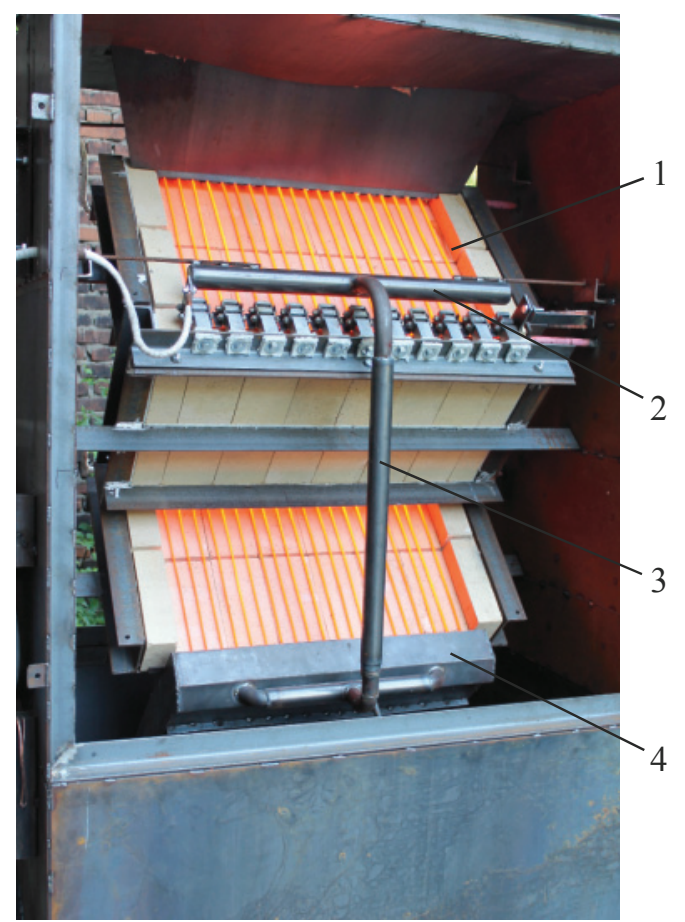

Рис. 5. Электрическая печь с дополнительным «нулевым» модулем: 1 - модуль со снятой термокрышкой; 2 всасыватель; 3 - воздуховод; 4 - «нулевой» модуль

Fig. 5. Electric furnace with an additional "zero» module: 1 is the module with a removed thermo-lid; 2 is the suction unit; 3 is the air duct; 4 is the "zero" module 
Опыты проводились на вермикулитовом конгломерате с содержанием флогопита песка и камней с частицами 1,5-5 мм при их совокупной массовой доле $\sim 18 \%$. Свойства сырья существенно отличались от свойств концентратов Ковдорского месторождения, оно обжигалось при более высоких температурах 800-1000 ${ }^{\circ} \mathrm{C}$, но минимальная плотность после вспучивания не опускалась ниже $223 \mathrm{\kappa г} / \mathrm{M}^{3}$ (типовой концентрат КВК-4 вспучивался до 80-98 кг $\left./ \mathrm{M}^{3}\right)$.

В табл. 1 приведены результаты экспериментов: температура нагревателей, средние по трем измерениям значения плотностей на входе $\rho_{\text {вх.ср }}$ и

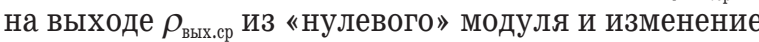
плотности вспученного материала после прохождения через «нулевой» модуль $\Delta \rho$.

По мере увеличения температуры разность насыпных плотностей уменьшается. Так, при температуре $801{ }^{\circ} \mathrm{C}$ разность плотностей равна $66 \mathrm{\kappa г} / \mathrm{m}^{3}$, при $T=890{ }^{\circ} \mathrm{C} \Delta \rho-29$ кг $/ \mathrm{m}^{3}$, а при минимальной плотности $223 \mathrm{\kappa} / \mathrm{M}^{3}$ разность плотностей $\Delta \rho$ составляет 9 кг $/ \mathrm{M}^{3}$.

таблица 1. Результаты экспериментов

Table 1. Experimental results

\begin{tabular}{|c|c|c|c|c|}
\hline \multirow{2}{*}{ 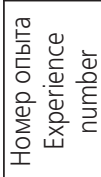 } & \multirow{2}{*}{$\begin{array}{c}\text { Средняя } \\
\text { температура } \\
\text { нагревателей, }{ }^{\circ} \mathrm{C} \\
\text { Average tempera- } \\
\text { ture of heaters, }{ }^{\circ} \mathrm{C}\end{array}$} & \multicolumn{2}{|c|}{$\begin{array}{c}\text { Плотность, кг } / \mathrm{M}^{3} \\
\text { Density, } \mathrm{kg} / \mathrm{m}^{3}\end{array}$} & \multirow{2}{*}{$\begin{array}{c}\text { Разность } \\
\text { плотностей } \\
\Delta \rho \\
\text { Difference of } \\
\text { densities } \Delta \rho\end{array}$} \\
\hline & & $\begin{array}{c}\rho_{\text {вх.ср }} \\
\text { на входе } \\
\text { at input }\end{array}$ & $\begin{array}{c}\rho_{\text {вых.ср }} \\
\text { на выходе } \\
\text { at output }\end{array}$ & \\
\hline 1 & 801 & 337 & 294 & 66 \\
\hline 2 & 826 & 323 & 272 & 44 \\
\hline 3 & 858 & 301 & 269 & 38 \\
\hline 4 & 890 & 279,5 & 245 & 29 \\
\hline 5 & 921 & 252 & 238 & 21 \\
\hline 6 & 953 & 345 & 229 & 11 \\
\hline 7 & 992 & 232 & 223 & 9 \\
\hline
\end{tabular}

В работе [14] получены результаты ранее выполненных экспериментов на опытно-промышленной печи без «нулевого» модуля.

Таблица 2. Концентрат КВК-4 Ковдорского месторождения

Table 2. Concentrate KVK-4 of Kovdorskoye deposit

\begin{tabular}{|c|c|c|c|}
\hline \multirow{2}{*}{$\begin{array}{l}\text { № модуля } \\
\text { (сверху } \\
\text { вниз) } \\
\text { Module No. } \\
\text { (top down) }\end{array}$} & \multicolumn{2}{|c|}{ 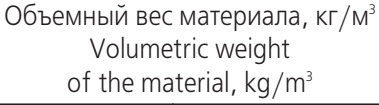 } & \multirow{2}{*}{$\begin{array}{c}\text { Изменение } \\
\text { объемного веса } \\
\text { на модуле } \Delta \gamma_{\mathrm{B}} \\
\mathrm{\kappa r} / \mathrm{M}^{3} \\
\text { Change in volumet } \\
\text { ric weight on the } \\
\text { module } \Delta \gamma_{\mathrm{B}}, \mathrm{kg} / \mathrm{m}^{3}\end{array}$} \\
\hline & $\begin{array}{c}\gamma_{\text {вх на входе }} \\
\text { в модуль } \\
\text { at the module } \\
\text { input }\end{array}$ & $\begin{array}{c}\gamma_{\text {вых }} \text { на выходе } \\
\text { из модуля } \\
\text { at the module } \\
\text { output }\end{array}$ & \\
\hline 1 & 540 & 126 & 414 \\
\hline 2 & 126 & 94 & 32 \\
\hline 3 & 94 & $\begin{array}{c}86 \\
\text { (на выходе печи) } \\
\text { (at the output } \\
\text { of the furnace) }\end{array}$ & 8 \\
\hline
\end{tabular}

Данные демонстрируют (табл. 2), что на третьем (нижнем) модуле изменение плотности вермикулита составляет всего $8 \mathrm{kг} / \mathrm{m}^{3}$. В новых опытах изменение плотности вспучиваемого продукта при его прохождении через «нулевой» модуль составляет 9 кг/ $\mathrm{m}^{3}$. Из сравнения виден эффект «нулевого» модуля: он способен заменить электрический модуль без снижения качества вспучивания и снижения температуры накала электронагревателей в оставшихся модулях.

Для трехмодульной печи отношение $\Delta \rho$ к среднему значению плотности по входу и выходу для КВК-4 (табл. 2):

$$
\rho_{\text {от }}=\frac{\Delta \rho}{0,5\left(\rho_{\text {вх }}+\rho_{\text {вых }}\right)}=\frac{8}{0,5(94+86)}=0,089 .
$$

Для вспучиваемого в новом эксперименте конгломерата данное отношение иное:

$$
\rho_{\text {от }}^{\prime}=\frac{9}{0,5(232+223)}=0,04,
$$

т. к. его конечная плотность существенно больше.

Если при $\rho_{\text {от }}=0,089$ снижение потребляемой мощности составляет треть от общей мощности $(30,3 \%)$, то при $\rho_{\text {от }}^{\prime}=0,04$ снижение, исходя из соответствующей пропорции, составит:

$$
0,04 \frac{33,3 \%}{0,089}=14,9 \% \text {. }
$$

Следовательно, использование дополнительного «нулевого» модуля в трехмодульной печи позволит снизить накал нагревателей и сэкономить около $15 \%$ энергии.

Очевидно, что эксергия, запасенная в крупных зернах вермикулита и частицах инертного материала к моменту их перехода из электрического модуля в «нулевой», действительно работает, трансформируясь в механическую энергию их структурообразования.

По мере удаления от минимальной плотности в область ее бо́льших значений (в зону не полного вспучивания продукта), эффективность «нулевого» модуля возрастает (табл. 1), но уменьшать потребляемую мощность за счет снижения качества вермикулита нецелесообразно. Однако, учитывая эту тенденцию, дополнительный модуль можно устанавливать не последним в цепи модулей обжига, a, например, предпоследним, чтобы еще больше повысить его эффективность. При этом последним будет типовой электрический модуль.

\section{Влияние охлаждения на прочность вспученного вермикулита}

Быстрое охлаждения вспученного вермикулита, как отмечалось выше, оказывает влияние на его прочность [15]. В других источниках информации, как в работе [15], нет конкретных данных по прочности, поэтому мы проверили опыты на промышленной печи зимой 2018 г. Вспученный вермикулит, выходивший из нижнего электрического модуля, рассыпали тонким слоем на фанерном листе и быстро (7-8 с) выносили на мороз.

Эксперименты по сравнению прочности проводились на стенде, работающем как поршень в ци- 
линдре. В табл. 3, 4 приведены значения сжимающего усилия $F$ на поршне, соответствующие им значения абсолютной деформации массива вермикулита $\Delta h$ в цилиндре, нормальное напряжение $\sigma$ (прочность в цилиндре) и относительная деформация массива $\varepsilon$.

Таблица 3. Нагрузка и деформация охлажденного вермикулита Table 3. Load and deformation of cooled vermiculite

Вермикулит Кокшаровского месторождения. Размерность 3,0 мм Vermiculite of the Koksharovskoye deposit. Dimension is $3,0 \mathrm{~mm}$

\begin{tabular}{|c|c|c|c|c|c|c|c|c|}
\hline$F, \mathrm{H}$ & 39,2 & 69,7 & 165,8 & 200,1 & 295,3 & 354,1 & 415,0 & 488,5 \\
\hline$\Delta h, \mathrm{M}$ & 0,008 & 0,009 & 0,016 & 0,017 & 0,022 & 0,024 & 0,026 & 0,032 \\
\hline$\sigma, \mathrm{H} / \mathrm{M}^{2}$ & 1032 & 1834 & 4363 & 5265 & 7771 & 9318 & 10921 & 12855 \\
\hline$\varepsilon_{,} \%$ & 4 & 4,5 & 8 & 8,5 & 11 & 12 & 13 & 16 \\
\hline
\end{tabular}

Таблица 4. Нагрузка и деформация неохлажденного вермикулита

Table 4. Load and deformation of non-cooled vermiculite

\begin{tabular}{|c|c|c|c|c|c|c|c|c|}
\hline \multicolumn{9}{|c|}{$\begin{array}{l}\text { Вермикулит Кокшаровского месторождения. Размерность } 3,0 \text { мм } \\
\text { Vermiculite of the Koksharovskoye deposit. Dimension is } 3,0 \mathrm{~mm}\end{array}$} \\
\hline$F, H$ & 39,2 & 69,7 & 165,8 & 200,1 & 295,3 & 354,1 & 415,0 & 488,5 \\
\hline$\Delta h, \mathrm{M}$ & 0,008 & 0,01 & 0,017 & 0,019 & 0,024 & 0,027 & 0,03 & 0, \\
\hline $5, \mathrm{H} / \mathrm{M}^{2}$ & 1032 & 1834 & 4363 & 5265 & 7771 & 9318 & 10921 & 12855 \\
\hline$\varepsilon, \%$ & 4 & 5 & 85 & 9.5 & 12 & 13,5 & 15 & 1 \\
\hline
\end{tabular}

Нормальное давление, вызывающее напряжение в материале при сжатии, и относительная деформация массива вермикулита в цилиндре определялись расчетом по формулам:

$$
\sigma=\frac{F}{S} \text { и } \varepsilon=\frac{\Delta h}{h} \cdot 100 \%,
$$

где $\sigma$ - нормальное давление; $S$ - площадь цилиндра; $\Delta h$ - абсолютная, и относительная $\varepsilon$ деформация проб вермикулита; $h$ - исходная высота пробы в цилиндре.

Ниже показаны графики зависимости прочности вермикулита в цилиндре от относительной деформации массива (рис. 6). Поскольку для сыпучих материалов прочность в цилиндре определяют при 10 и $20 \%$-й деформации, сравним прочность охлажденного $\left(\sim 6550 \mathrm{H} / \mathrm{m}^{2}\right)$ и неохлажденного $\left(\sim 5500 \mathrm{H} / \mathrm{m}^{2}\right)$ материала при деформации массива $10 \%$. Эффект охлаждения очевиден: увеличение прочности составляет $19,1 \%$.

Однако условия охлаждения в данном опыте и в производственных условиях - в охладителе технологического комплекса, отличаются. Поэтому необходимо моделирование этого процесса. Особенность задачи состоит в том, что вермикулитовые концентраты имеют разные размеры зерен. Так, концентраты Ковдорского месторождения делятся на марки крупности: КВК-0,5; КВК- 1 ; КВК2 ; КВК-4 и КВК-8, и в каждой группе есть частицы, отличающиеся размерами в 6-9 раз. С учетом этого повышение качества обжига и охлаждения вермикулита может быть достигнуто за счет предварительного фракционирования сырья.

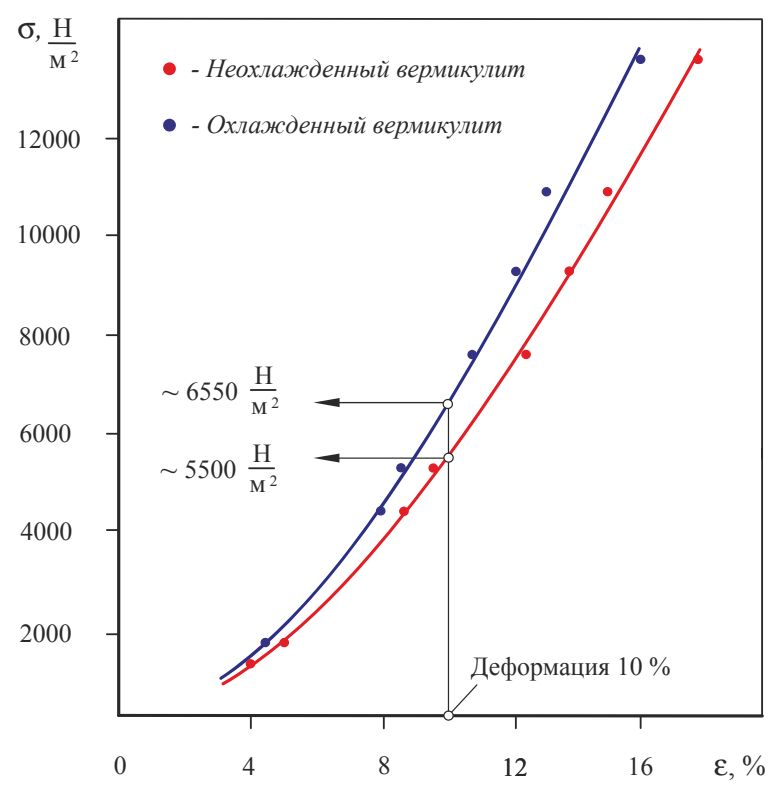

Рис. 6. Зависимость прочности в цилиндре от относительной деформации массива

Fig. 6. Dependence of the strength in a cylinder on the relative deformation of an array

Время распространения температуры от центров чешуек вспученного зерна к его периферии, где потоком воздуха поддерживается постоянная температура $\sim 20^{\circ} \mathrm{C}$ (рис. 7) можно рассчитать исходя из температуропроводности вещества $\chi$, характеризующего скорость выравнивания температуры в неравновесных тепловых процессах [16].

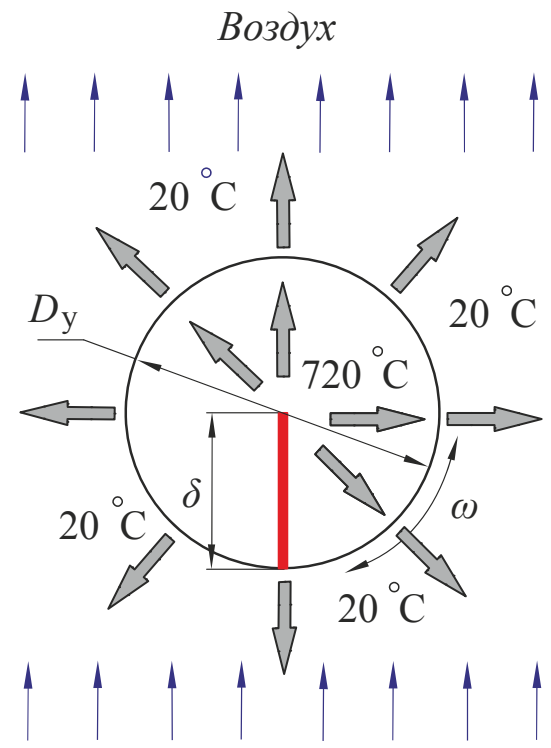

Рис. 7. Схема к моделированию температуропроводности вермикулитовых зерен

Fig. 7. Scheme for modeling thermal conductivity of vermiculite grains

Вспученный вермикулит обладает чешуйчатой структурой и, как следствие, выраженной анизотропией свойств. Чешуйки чередуются со щеле- 
видными порами, поэтому модель температуропроводности не учитывает тепловой поток в поперечном направлении. Выделим из вспученного зерна чешуйку с условным диаметром $D_{y}$ (рис. 7) и определим время выравнивания температуры от ее центра к краям.

Сделаем ряд допущений:

- пусть перед входом в охладитель все процессы, связанные с теплопереносом, дегидратацией и вспучиванием, завершены;

- при охлаждении температура на краях чешуек $\left(\sim 20^{\circ} \mathrm{C}\right)$ возникает мгновенно и поддерживается постоянной за счет непрерывного обтекания зерен потоком воздуха;

- при завершении охлаждения полного выравнивания температур в центрах чешуек и на их периферии не происходит;

- температуропроводность в любой точке чешуйки одинакова;

- в соседних чешуйках идут аналогичные процессы, и теплообмена между ними нет;

- при охлаждении взаимный перенос лучистой энергии между зернами отсутствует.

Зависимость отношения разности температур $\Delta T_{1}$ на периферии чешуйки и в ее центре в конце охлаждения на выходе из охладительного устройства и в начале охлаждения $\Delta T_{2}$ на входе в него от времени $t$ описывается уравнением [17]:

$$
\frac{\Delta T_{1}}{\Delta T_{2}}=\frac{T_{a}-T_{b}}{T_{c}-T_{d}}=1,03 e^{-2,47 \frac{\chi t}{\delta^{2}}},
$$

где $T_{a}$ - температура в центре чешуйки в конце охлаждения, ${ }^{\circ} \mathrm{C} ; \mathrm{T}_{b}$ - температура на периферии зерна в конце охлаждения, ${ }^{\circ} \mathrm{C} ; T_{c}$ - температура на периферии в начале охлаждения, ${ }^{\circ} \mathrm{C} ; T_{d}$ - температура в глубине зерен в начале охлаждения, ${ }^{\circ} \mathrm{C} ; \chi-$ коэффициент температуропроводности, $\mathrm{m}^{2} / \mathrm{c} ; \delta-$ фрагмент чешуйки, равный ее условному радиусу.

Возможность применения формулы (1) можно проверить условием [17]:

$$
\chi \frac{t}{\delta^{2}}>0,06 \text {. }
$$

Время $t$ не известно, но его ожидаемое значение $t_{0}$ можно найти для случая, когда температуры на концах выделенного фрагмента не влияют на время их выравнивания [16]:

$$
t_{\mathrm{o}}>0,06 \frac{\delta^{2}}{\chi} \text {. }
$$

Для зерна с условным диаметром $D_{y}=4,0$ мм $\delta$ равна $2,0 \cdot 10^{-3}$ м. Для нахождения ожидаемого времени и проверки условия (2) определим коэффициент температуропроводности [16]:

$$
\chi=\frac{\lambda}{\rho c},
$$

где $\lambda$ - коэффициент теплопроводности; $\rho$ - истинная плотность; $c$ - удельная теплоемкость.

Пористость зерен определяется щелевыми порами, а «тело» чешуйки является сплошным и ко- эффициент 1? для нее можно принять как для вермикулита или флогопита-сырца, равным в направлении плоскостей спайности $\sim 5,1 \mathrm{Bт} / \mathrm{м}^{\circ}{ }^{\circ} \mathrm{C}$ [18]. Плотность по этим же соображениям принимаем $2300 \mathrm{\kappa г} / \mathrm{M}^{3}$ [19], но с учетом выхода большей части гидратной воды ( 18-20\% [20]) уточненное значе-

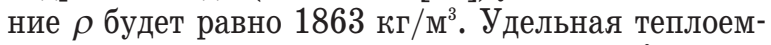
кость $c$ слюды-сырца при температуре $20{ }^{\circ} \mathrm{C}$ равна $\sim 80$ Дж/кг ${ }^{\circ} \mathrm{C}$, а в интервале температур от 20 до $720{ }^{\circ} \mathrm{C}$ ее можно принять примерно равной 930 Дж/кг. ${ }^{\circ} \mathrm{C}[21]$.

Расчет по формулам (3), (4) дает значение $\chi=2,9 \cdot 10^{-6} \mathrm{M}^{2} /$ с и $t_{0}=1,38$ с. Проверка условия (2) подтверждает его выполнение:

$$
1,0005>0,06 \text {. }
$$

Вернемся к формуле (1) и зададим температуры. Если полного выравнивания температур $T_{a}$ и $T_{b}$ к моменту выхода вспученных зернен из охладителя не произойдет, то зададим остаточную температуру $T_{a}$ в центре чешуйки в конце охлаждения, например, $50{ }^{\circ} \mathrm{C}$, а температуру $T_{b}-$ на периферии в конце охлаждения, равной температуре воздуха $20{ }^{\circ} \mathrm{C}$.

Пусть температура $T_{c}$ на периферии в начале охлаждения равна $720^{\circ} \mathrm{C}$, а в глубине зерен в начале охлаждения $T_{d}$ меньше $T_{c}$, но достаточна для полной дегидратации и вспучивания зерна в печи и равна $\sim 500{ }^{\circ} \mathrm{C}$. Тогда левая часть выражения (1) будет равна 0,12 и его можно переписать в виде:

$$
0,12=1,03 e^{-2,47 \frac{\chi t}{\delta^{2}}} .
$$

После преобразований получим:

$$
e^{2,47 \frac{\chi t}{\delta^{2}}}=8,58
$$

и окончательно:

$$
e^{1,79 t}=8,58 .
$$

Степень $1,79 t$ основания натурального логарифма $e$ для удовлетворения условию (5) должна быть равна 2,15, поэтому:

$$
1,79=2,15 \text {. }
$$

Из выражения (6) получаем время выравнивания 1,2 с, при котором обеспечивается охлаждение зерен потоком воздуха с температурой $20{ }^{\circ} \mathrm{C}$. Проводя аналогичные расчеты для зерен с другими условными диаметрами, получим значения времени выравнивания $t$, соответствующие этим $D_{y}$ (табл. 5).

Полученные данные проиллюстрированы графиком зависимости времени выравнивания температур в центре и на периферии вспученных зерен от их условного диаметра (рис. 8). На нем хорошо видно, что даже внутри одной размерной группы вермикулитового концентрата марки КВК-4 время выравнивания температур самых крупных вспученных зерен ( $D_{y}=7,5$ мм) в 25 раз превышает время выравнивания температур самых мелких из них $\left(D_{y}=1,5 \mathrm{~mm}\right)$.

Для зерен с $D_{y}=7,5$ мм время выравнивания составляет 4,22 с и это слишком много. Охлаждение 
должно идти быстрее, чтобы остановить в глубинных слоях зерен все физико-химические процессы, только в этом случае вспученные зерна не потеряют прочность.

Таблица 5. Время выравнивания температур для зерен вермикулита

Table 5. Time of temperature equalization for vermiculite grains

\begin{tabular}{|c|c|c|c|c|}
\hline $\begin{array}{l}\text { Условный ди- } \\
\text { аметр } D_{y,} \text { мм } \\
\text { Nominal dia- } \\
\text { meter } D_{y}, \mathrm{~mm}\end{array}$ & $\begin{array}{l}\text { Ожидае- } \\
\text { мое } \\
\text { время } t_{0}, c \\
\text { Expected } \\
\text { time } t_{0}, s\end{array}$ & $\begin{array}{c}\text { Выполнение } \\
\text { условия (2) } \\
\text { Fulfillment } \\
\text { of condition } \\
\text { (2) }\end{array}$ & $\begin{array}{c}\text { Показатель } \\
\text { степени } \\
\text { Exponent } \\
2,47 \chi t / \delta^{2}\end{array}$ & $\begin{array}{c}\text { Время вы- } \\
\text { равнивания } \\
t, c \\
\text { Equalization } \\
\text { time } t, s\end{array}$ \\
\hline 1,5 (т. a) & 0,19 & \multirow{7}{*}{$\begin{array}{c}\text { Выполняется } \\
\text { Fulffilled }\end{array}$} & $12,7 t$ & 0,17 \\
\hline 2,5 (т. b) & 0,54 & & $4,58 t$ & 0,46 \\
\hline 3,5 (т. C) & 1,056 & & $2,34 t$ & 0,92 \\
\hline 4,5 (т. d) & 1,75 & & $1,41 t$ & 1,54 \\
\hline 5,5 (т. e) & 2,6 & & $0,91 t$ & 2,39 \\
\hline 6,5 (т. f) & 3,77 & & $0,67 t$ & 3,21 \\
\hline 7,5 (т. g) & 4,85 & & $0,51 t$ & 4,22 \\
\hline
\end{tabular}

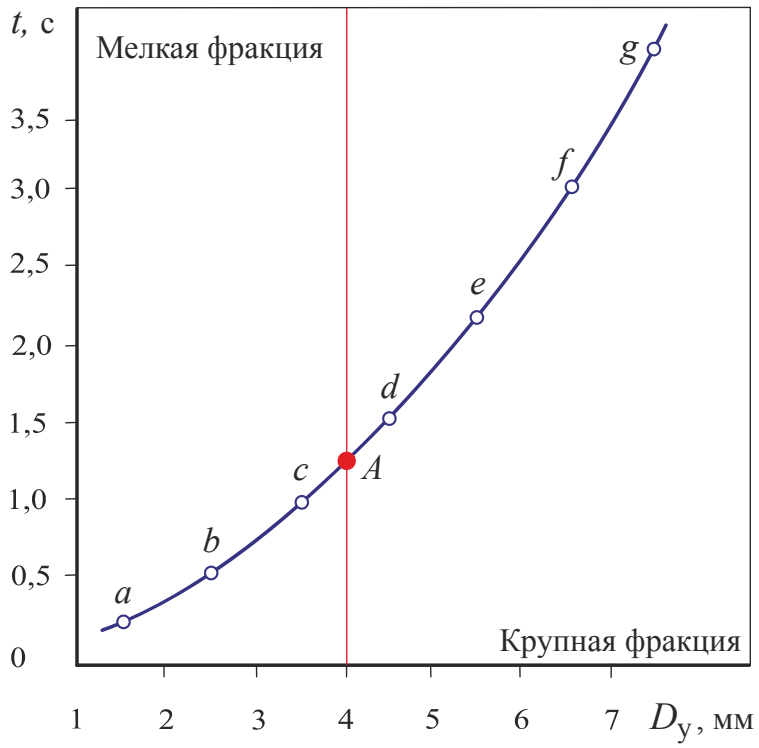

Рис. 8. Зависимость времени выравнивания температур от условного диаметра зерен вермикулита

Fig 8. Dependence of temperature equalization time on the nominal diameter of vermiculite grains

Из литературных источников известно, что при нагреве до $150{ }^{\circ} \mathrm{C}$ вермикулит гарантировано не изменяет своей исходной структуры [22]: из него выходит только свободная вода. Вспучивание начинается лишь при нагреве более $270{ }^{\circ} \mathrm{C}$ и сопровождается выходом химически связанной воды, структурообразованием и протеканием химических реакций [22].

Если задать остаточную температуру $T_{a}$ в центре чешуек крупных зерен в конце охлаждения $150{ }^{\circ} \mathrm{C}$, то можно утверждать, что изменений физических свойств - прочности, хрупкости и упругости, уже не будет.

Определим временя охлаждения $\left(t_{x}\right)$, используя формулу (1). Ее левая часть будет изменяться, и уравнение (1) примет вид:

$$
x_{i}=1,03 e^{-2,47 \frac{\chi t}{\delta^{2}}},
$$

где $x_{i}$ - это отношение $\Delta T_{1} / \Delta T_{2}$, которое определится при подстановке в выражение (7) нескольких значений остаточной после обжига температуры $T_{a}$ $\left({ }^{\circ} \mathrm{C}\right): 50,75,100,125,150$.

Подстановка этих значений и использование вышеприведенного алгоритма расчета времени позволяют рассчитать время охлаждения крупных зерен концентрата КВК-4 (табл. 6).

Таблица 6. Время охлаждения зерен с условными диаметрами 7,5; 6,5 и 5,5 мм

Table 6. Cooling time of grains with conditional diameters 7,$5 ; 6,5$ и $5,5 \mathrm{~mm}$

\begin{tabular}{|c|c|c|c|}
\hline \multirow{2}{*}{$\Delta T_{1},{ }^{\circ} \mathrm{C}$} & \multicolumn{3}{|c|}{ Bpems $t_{x,} \mathrm{C} /$ Time $_{x_{x}} \mathrm{~s}$} \\
\cline { 2 - 4 } & $\begin{array}{c}D_{y}=7,5 \mathrm{MM}, \\
\delta=3,75 \mathrm{MM}\end{array}$ & $\begin{array}{c}D_{y}=6,5 \mathrm{MM}, \\
\delta=3,25 \mathrm{MM}\end{array}$ & $\begin{array}{c}D_{y}=5,5 \mathrm{MM}, \\
\delta=2,75 \mathrm{MM}\end{array}$ \\
\hline 30 & 4,2 & 3,21 & 2,39 \\
\hline 55 & 3,0 & 2,26 & 1,62 \\
\hline 80 & 2,29 & 1,72 & 1,23 \\
\hline 105 & 1,8 & 1,37 & 0,98 \\
\hline 130 & 1,33 & 1,0 & 0,72 \\
\hline
\end{tabular}

Как видно из табл. 6, для крупных зерен с $D_{y}=7,5$ мм при разности температур в центре и на краях $T_{a}-T_{b}=150-20=130{ }^{\circ} \mathrm{C}$ достаточным будет время охлаждения $t_{x}=1,33 \mathrm{c}$, что вполне реализуемо в охладителе, а для зерен меньшего размера оно уменьшается до 1,0 и 0,72 с.

На рис. 9 показаны зависимости времени охлаждения крупных зерен от разности температур в центре и периферии чешуек. Они имеют общую тенденцию $\mathrm{k}$ снижению $t_{x}$ при увеличении $\Delta T_{1}$. Но важно то, что если для всего массива вспученных зерен задать время охлаждения по максимальным зернам, например - 1,4 c, то более мелкие зерна будут в разной степени переохлажденными с температурами внутри $20,30,40{ }^{\circ} \mathrm{C}$. Крупные зерна, находясь в едином массиве с мелким, быстро сравняются с ними за счет кондуктивного теплообмена.

Время охлаждения самого крупного из вспученных зерен $t_{x}$ должно быть равно времени его движения в канале - 15 охладителя (рис. 1).

Падение одиночного зерна можно описать дифференциальным уравнением:

$$
m \ddot{x}=m g-\alpha\left(v_{0}+\dot{x}\right)^{2},
$$

где $m$ - масса зерна, кг; $\dot{x}$ - скорость его падения, $\mathrm{M} / \mathrm{c} ; v_{0}$ - скорость встречного потока воздуха, м/с; $\alpha$ - коэффициент сопротивления, кг/м.

Решение уравнения (8) является тривиальным и позволяет найти зависимость перемещения зерна от времени падения $\tau$, с. Приравнивая время охлаждения и падения $t_{x}=\tau$, несложно найти необходимую высоту канала - 15 охладителя.

Отметим, что процесс охлаждения зерен полностью завершается не в охладителе, а в продуктопроводе - 19 (рис. 1). 


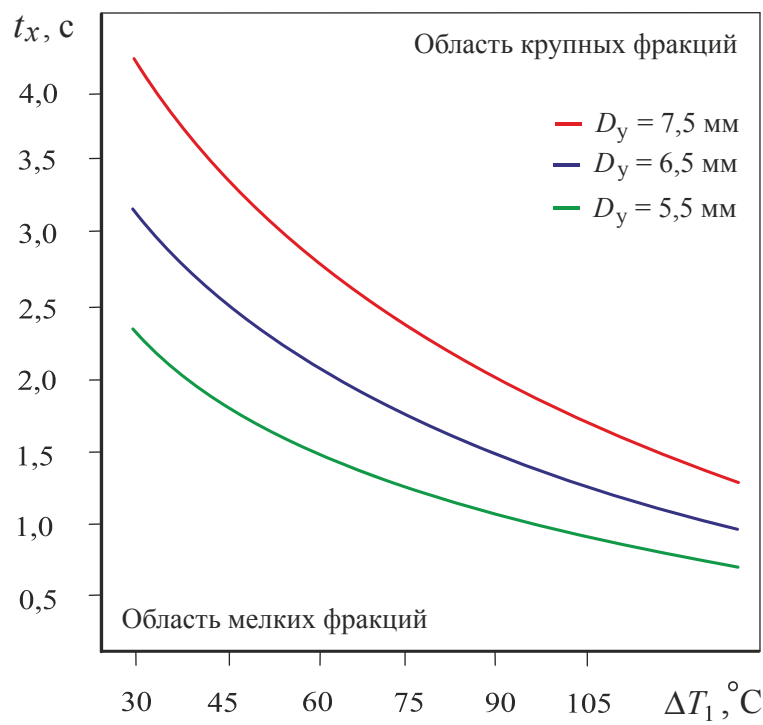

Рис. 9. Зависимости времени охлаждения крупных зерен от разности температур в центре и периферии чешуек

Fig. 9. Dependences of cooling time of large grains on the temperature difference in the center and the periphery of the flakes

\section{Выделение мелкодисперсного вермикулита}

Очищенный от мелких частиц вермикулит (рис. 3, в) обладает меньшей плотностью, что повышает его качество, но и сами эти частицы являются ценным вторичным продуктом, который находит применение в производстве строительных и огнеупорных материалов [1]. На рис. 10 показаны два типа вторичного продукта - мелкочешуйчатый (a) и мелкодисперсный (б).

Отбор этих частиц идет не только в охладителе через канал - 13 , но и через всасыватели - 4 , которые уносят их вместе с горячим воздухом при его прокачке по тепловым камерам - 5, 6 «нулевого» модуля (рис. 2), а накопление происходит в бункере-осадителе - 17 (рис. 1).

Теория отделения мелкодисперсного материала уже рассматривалась в работах авторов, поэтому здесь мы приведем только некоторые опытные данные.

Опыт эксплуатации электрических модульноспусковых печей показывает, что через всасыватели собирается около 45 \% вторичного продукта, остальное выходит из печи вместе с вермикулитом [23]. Поэтому в рассматриваемом технологическом комплексе охладитель выполняет и вторую функцию - отбор чешуйчатых и мелкодисперсных частиц.

На рис. 11 приведены зависимости суточного объема вторичного продукта $V_{\text {вп }}$ от объема вспученного вермикулита $V_{\text {в }}$ при 45-процентном сборе по Ковдорскому (КВК) и Татарскому (КВТ) сырью.

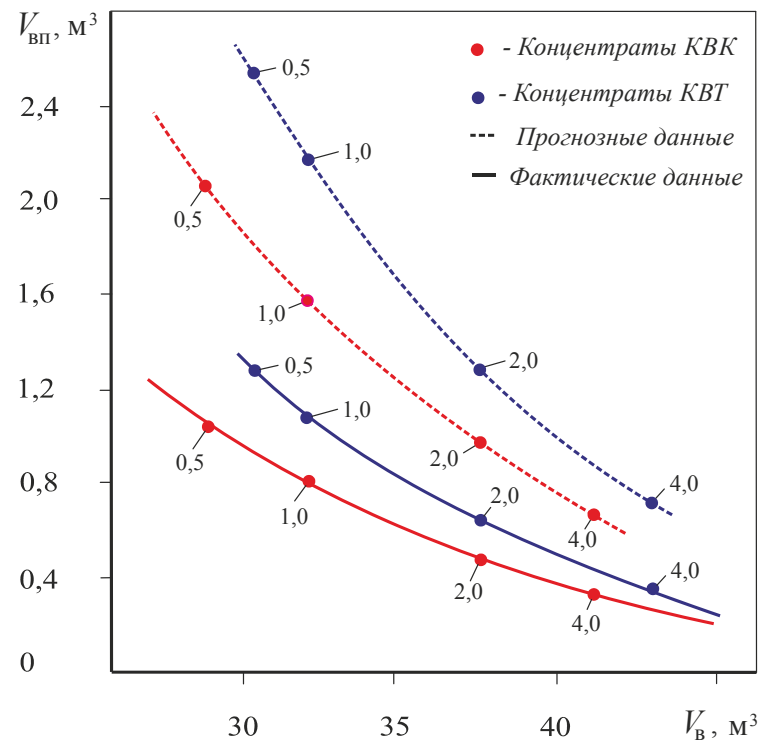

Рис. 11. Зависимость суточного объема вторичных продуктов от объема очищенного вермикулита

Fig. 11. Dependence of a daily volume of secondary products on a volume of purified vermiculite

C учетом производительности опытно-промышленной печи по различным размерным группам концентратов были рассчитаны суточные объемы основного материала, соответствующие объемам вторичных продуктов. При известной массовой доле выделенного вермикулита можно рассчитать снижение его плотности $\gamma_{\text {в }}$ за счет выведения из общего массива вторичных продуктов:

$$
\gamma_{\mathrm{B}}^{\prime}=\gamma_{\mathrm{B}}-\frac{m}{V_{\mathrm{B}}} .
$$

Используя среднестатистические данные по объемному весу вспученных вермикулитовых про-

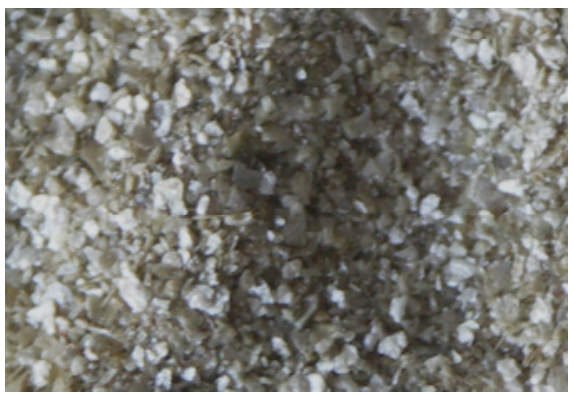

$a / a$

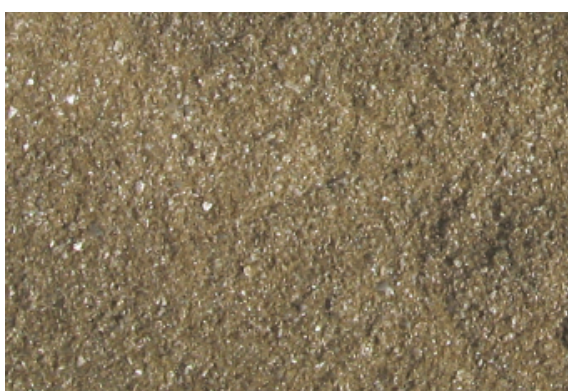

$\sigma / b$

Рис. 10. Вермикулит: а) чешуйчатый; б) мелкодисперсный

Fig. 10. Vermiculite: a) scaly; b) finely dispersed 
дуктов [1], в результате вычислений по формуле (9) получаем плотность очищенного продукта $\gamma_{\text {в }}^{\prime}$, кг/ $\mathrm{M}^{3}$ (выборочно):

- Ковдорского:

$\gamma_{\mathrm{B}(\mathrm{KBT}-1)}^{\prime}=115,1 \mathrm{\kappa \Gamma} / \mathrm{M}^{3}$;

$\gamma_{\mathrm{B}(\mathrm{KBT}-2)}^{\prime \prime}=99,68 \mathrm{\kappa \Gamma} / \mathrm{M}^{3}$;

$\gamma_{\mathrm{B}(\mathrm{KBT}-4)}^{\prime}=86,6 \mathrm{\kappa \Gamma} / \mathrm{M}^{3}$;

- Татарского: $\gamma$

${ }_{\text {в (КВТ-1) }}=119,0 \mathrm{K \Gamma} / \mathrm{M}^{3}$;

$\gamma_{\mathrm{B}(\mathrm{KBT}-2)}^{\prime}=98,93 \mathrm{\kappa г} / \mathrm{M}^{3}$.

После выделения вторичных продуктов достиг-

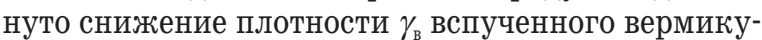
лита до $3 \%$ для крупных концентратов и до 8,5 \% для мелких.

В новом технологическом комплексе за счет сбора вторичных продуктов через систему охлаждения можно прогнозировать его увеличение до 90 \% . На рис. 11 показаны графики, примерно соответствующие прогнозу.

\section{Заключение}

Технологический комплекс для переработки вермикулитовых концентратов и конгломератов характеризуется целым рядом новых технический решений, направленных на экономию электроэнергии, повышение качества вспученного вермикулита, получение вторичных целевых продуктов - чешуйчатого и мелкодисперсного вермикулита, а также на выделение инертного материала

\section{СПИСОК ЛИТЕРАТУРЫ}

1. Fuks L., Herdzik-Koniecko I. Vermiculite as a potential component of the engineered barriers in low- and medium-level radioactive waste repositories // Applied Clay Science. - 2018. V. 161. - P. 139-150.

2. Rashad A.M. Vermiculite as a construction material - a short guide for Civil Engineer // Construction and Building Materials. 2016. - V. 125. - P. 53-62.

3. Hombostel C. Construction Materials: Types, Uses, and Applications. - New York: John Wiley \& Sons, Inc., 1991. - 1040 p.

4. Mucahit Sutcun. Influence of expanded vermiculite on physical properties and thermal conductivity of clay bricks / Ceramics International. - 2015. - V. 41. - P. 2819-2827.

5. Kariya J., Ryu J., Kato Y. Development of thermal storage material using vermiculite and calcium hydroxide // Applied Thermal Engineering. - 2016. - V. 94. - P. 186-192.

6. Industrial minerals \& rocks: commodities, markets, and used / Ed. by J.E. Kogal. - Littleton: Society for Mining, Metallurgy, and Exploration, Inc., 2006. -1529 p.

7. Fan Ding et al. Tuning wettability by controlling the layer charge and structure of organo-vermiculites // Journal of Industrial and Engineering Chemistry. - 2018. - V. 57. - P. 304-312.

8. Sevim Ișçi. Intercalation of vermiculite in presence of surfactants // Applied Clay Science. - 2017. - V. 146. - P. 7-13.

9. Sevim İşçi, Yavuz İşci. Characterization and comparison of thermal \& mechanical properties of vermiculite polyvinylbutyral nanocomposites synthesized by solution casting method // Applied Clay Science. - 2017. - V. 151. - P. 189-193.

10. Malandrino M. et al. Adsorption of heavy metals on vermiculite: influence of $\mathrm{pH}$ and organic ligands // J. Colloid Interface Sci. 2006. - V. 299. - P. 537-546.

11. Bryanskikh T.V., Kokourov D.V. Energy efficiency of electric furnaces with movable floor in firing of vermiculite concentrates (песка, каменистого гранулята и др.), который в случае переработки, например, вермикулит-сунгулитовых конгломератов, также может быть ценным материалом.

Усовершенствованная модульно-спусковая печь с так называемым «нулевым» неэлектрическим модулем, работающим за счет эксергии вермикулитовых зерен и частиц инертного материала, позволяет при той же производительности экономить до $15 \%$ электроэнергии и довести удельную энергоемкость обжига до 170-175 мДж/ $\mathrm{m}^{3}$ [24].

Система охлаждения обеспечивает повышение прочности вспученных зерен на 19,1 \% при одновременном увеличении почти до $90 \%$ (против $45 \%$ ) выделения вторичных целевых продуктов чешуйчатого и мелкодисперсного вермикулита.

Выведение вторичных продуктов и инертного материала значительно снижает насыпную плотность основного целевого продукта, а этот показатель является главным показателем качества.

Следующим этапом в работе по совершенствованию такого технологического комплекса будет привязка к нему новой электрической печи с подвижной подовой платформой, обладающей удельной энергоемкостью обжига вермикулита

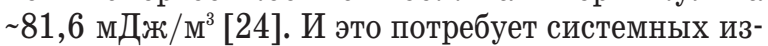
менений в конструкции комплекса без потерь всех достигнутых результатов.

of different size groups // Refractories and Industrial Ceramics Refractories and Industrial Ceramics. - 2017. - V. 58. P. 368-373. D0I: $10.1007 / \mathrm{s} 11148-017-0113-0$.

12. Figueiredo $\mathrm{S}$. The influence of acid treatments over vermiculite based material as adsorbent for cationic textile dyestuffs // Chemosphere. - 2016. - V. 153. - P. 115-129.

13. Mouzdahir Y. et al. Synthesis of nano-layered vermiculite of low density by thermal treatment // Powder Technol. - 2009. - V. 189. - P. 2-5.

14. Нижегородов А.И., Звездин А.В. Энерготехнологические агрегаты для переработки вермикулитовых концентратов. - Иркутск: Изд-во ИРНИТУ, 2015. - 250 с.

15. Кальянов Н.Н., Мерзляк А.Н. Вермикулит и перлит - пористые заполнители для теплоизоляционных изделий и бетонов. - М.: Гос. изд-во по строительству, архитектуре и строительным материалам, $1961 .-154 \mathrm{c.}$

16. Китайгородский А.И. Введение в физику. - М.: Наука, 1973 $688 \mathrm{c.}$

17. Расчет времени для выравнивания температуры / Вуневеpe.py. 2018. URL: http://vunivere.ru/work27956/page2 (дата обращения 20. 05.2018).

18. Слюда. Теплопроводность // Энциклопедия по машиностроению XXL. Оборудование, материаловедение, механика. URL: http:// mash-xxl.info/info/452250/ (дата обращения 20.05.2018).

19. Слюда. Сравнительные свойства природных и синтетических слюд. 2006-2015. URL: http://cniga.com.ua/index.files/sluda.htm (дата обращения 20. 05. 2018).

20. Nizhegorodov A.I., Zvezdin A.V. Study of an electric furnaces physical model for firing vermiculite with a «zero» module // Refractories and Industrial Ceramics. - 2016. - V. 57. № 3. P. 246-251. DOI: $10.1007 / \mathrm{s} 11148-016-9961-2$.

21. Методические рекомендации по применению классификации запасов к месторождениям слюды. - М.: Министерство природных ресурсов РФ, 2005. - 41 с. 
22. Производство и применение вермикулита / под ред. проф. Н.А. Попова. - М.: Стройиздат, 1964. - 128 с.

23. Nizhegorodov A.I. Theory and practical use of modular-pouring electric furnaces for firing vermiculite // Refractories and Industrial Ceramics. - 2015. - V. 56. - № 4. - P. 361-365.
24. Испытания новой альтернативной электрической печи для обжига вермикулитовых концентратов / А.И. Нижегородов, Т.Б. Брянских, А.Н. Гаврилин, Б.Б. Мойзес и др. // Известия Томского политехнического университета. Инжиниринг георесурсов. - 2018. - T. 329. - № 4. - С. 142-153.

Поступила 28.08.2018 2.

\section{Информация об авторах}

Нижегородов А.И., доктор технических наук, профессор кафедры строительных, дорожных машин и гидравлических систем Иркутского национального исследовательского технического университета.

Гаврилин A.Н., кандидат технических наук, доцент отделения материаловедения Инженерной школы новых производственных технологий Национального исследовательского Томского политехнического университета.

Мойзес Б.Б., кандидат технических наук, доцент отделения контроля и диагностики Инженерной школы неразрушающего контроля и безопасности Национального исследовательского Томского политехнического университета.

$\boldsymbol{K} у в ш и н о в ~ \boldsymbol{K} . \boldsymbol{A} .$, старший преподаватель отделения материаловедения Инженерной школы новых производственных технологий Национального исследовательского Томского политехнического университета. 


\title{
TECHNOLOGICAL COMPLEX FOR PROCESSING VERMICULITE CONCENTRATES AND CONGLOMERATES
}

\author{
Anatoly I. Nizhegorodov', \\ nastromo_irkutsk@mail.ru
}

Alexey N. Gavrilin², tom-gawra@@list.ru

Boris B. Moyzes², mbb@tpu.ru

\author{
Kirill A. Kuvshinov \\ kuvshinov@tpu.ru \\ 1 Irkutsk National Research Technical University, \\ 83, Lermontov Avenue, Irkutsk, 664074, Russia. \\ ${ }^{2}$ National Research Tomsk Polytechnic University, \\ 30, Lenin Avenue, Tomsk, 634050, Russia.
}

Relevance. Vermiculite and vermiculite-based materials have found wide application in many spheres of life: industry, environmental management, fire safety, etc. That's why, the solution of problems related to the improvement of technological processes for heat treating of vermiculite concentrates and conglomerates, especially in the aspect of obtaining high-quality expanded vermiculite, is relevant. The electric modular-trigger furnaces are the foundation for assurance of the resource efficiency of technological processes for expanded vermiculite production. Therefore, the consideration of issues of developing new electric modular-trigger furnaces designs and technological complexes based on them are relevant.

The aim of the research is to develop the technological complex for vermiculite processing, study of specified technological processes and evaluation of their effectiveness.

The research methods are based on the analysis of information sources in the field of research, the synthesis of constructive and technological solutions, parametric and functional description, experimental studies.

Results. The authors have developed a technological complex for processing vermiculite concentrates and conglomerates, taking into account the requirements of energy saving, improving the quality of expanded vermiculite and secondary target products. The modular-trigger furnace was improved. The furnace allows saving up to $15 \%$ of electric power at the same capacity. It was proved that the proposed cooling system provides an increase of the strength of the expanded grains by $19,1 \%$, an increase to almost $90 \%$ of the release of secondary target products. The main quality indicator - decrease in the poured density of the main target product due to removal of secondary products and inert material - is significantly increased. The authors forecast further works in the aspect of developing a new electric furnace with a movable bottom platform, which has a specific energy capacity for vermiculite heat treating of about $81,6 \mathrm{~mJ} / \mathrm{m}^{3}$.

\section{Key words:}

Technological complex, electric modular-trigger furnace, non-electric "zero» module, exergy, cooling system, separation system, vermiculite strength, secondary target products, inert material, thermal conductivity.

\section{REFERENCES}

1. Fuks L., Herdzik-Koniecko I. Vermiculite as a potential component of the engineered barriers in low- and medium-level radioactive waste repositories. Applied Clay Science, 2018, vol. 161, pp. $139-150$.

2. Rashad A.M. Vermiculite as a construction material - a short guide for Civil Engineer. Construction and Building Materials, 2016, vol. 125 , pp. 53-62.

3. Hombostel C. Construction Materials: Types, Uses, and Applications. New York, John Wiley \& Sons, Inc., 1991. 1040 p.

4. Mucahit Sutcun. Influence of expanded vermiculite on physical properties and thermal conductivity of clay bricks. Ceramics International, 2015, vol. 41, pp. 2819-2827.

5. Kariya J., Ryu J., Kato Y. Development of thermal storage material using vermiculite and calcium hydroxide. Applied Thermal Engineering, 2016, vol. 94, pp. 186-192.

6. Industrial minerals \& rocks: commodities, markets, and used. Ed. by J.E. Kogal. Littleton, Society for Mining, Metallurgy, and Exploration, Inc., 2006. 1529 p.
7. Fan Ding. Tuning wettability by controlling the layer charge and structure of organo-vermiculites. Journal of Industrial and Engineering Chemistry, 2018, vol. 57, pp. 304-312.

8. Sevim İşci. Intercalation of vermiculite in presence of surfactants. Applied Clay Science, 2017, vol. 146, pp. 7-13.

9. Sevim Isçi, Yavuz Ișçi. Characterization and comparison of thermal \& mechanical properties of vermiculite polyvinylbutyral nanocomposites synthesized by solution casting method. Applied Clay Science, 2017, vol. 151, pp. 189-193.

10. Malandrino M. Adsorption of heavy metals on vermiculite: influence of $\mathrm{pH}$ and organic ligands. J. Colloid Interface Sci., 2006, vol. 299 , pp. 537-546.

11. Bryanskikh T.V., Kokourov D.V. Energy efficiency of electric furnaces with movable floor in firing of vermiculite concentrates of different size groups. Refractories and Industrial Ceramics, 2017, vol. 58, pp. 368-373. DOI: 10.1007/s11148-017-0113-0.

12. Figueiredo $S$. The influence of acid treatments over vermiculite based material as adsorbent for cationic textile dyestuffs. Chemosphere, 2016, vol. 153, pp. 115-129. 
13. Mouzdahir Y. Synthesis of nano-layered vermiculite of low density by thermal treatment. Powder Technol., 2009, vol. 189, pp. 2-5.

14. Nizhegorodov A.I., Zvezdin A.V. Energotekhnologicheskie agregaty dlya pererabotki vermikulitovykh kontsentratov [Energytechnology units for processing vermiculite concentrates]. Irkutsk, IrNITU Press, 2015. 250 p.

15. Kalyanov N.N., Merzlyak A.N. Vermikulit i perlit - poristye zapolniteli dlya teploizolyatsionnykh izdeliy i betonov [Vermiculite and perlite-porous aggregates for heat-insulating products and concrete]. Moscow, State Publ. house in building, architecture and constructing materials, $1961.154 \mathrm{p}$.

16. Kitaygorodskiy A.I. Vvedenie $v$ fiziku [Introduction into physics]. Moscow, Nauka Publ., 1973. 688 p.

17. Raschet vremeni dlya vyravnivaniya temperatury [The calculated time for temperature equalization]. Vunevere.ru. 2018. Available at: http://vunivere.ru/work27956/page2 (accessed 20 May 2018).

18. Slyuda. Teploprovodnost [Mica. Thermal conductivity]. Entsiklopediya po mashinostroeniyu XXL. Oborudovanie, materialovedenie, mekhanika [Encyclopedia of mechanical engineering XXL. Equipment, materials science, mechanics]. Available at: http://mash-xxl.info/info/452250/ (accessed 20 May 2018).

19. Slyuda. Sravnitelnye svoystva prirodnykh i sinteticheskikh slyud [Mica. Comparative properties of natural and synthetic mica].
2006-2015. Available at: http://cniga.com.ua/index.files/sluda.htm (accessed 20 May 2018).

20. Nizhegorodov A.I., Zvezdin A.V. Study of an electric furnaces physical model for firing vermiculite with a «zero» module. Refractories and Industrial Ceramics, 2016, vol. 57, no. 3, pp. 246-251. DOI: 10.1007/s11148-016-9961-2.

21. Metodicheskie rekomendatsii po primeneniyu klassifikatsii zapasov $k$ mestorozhdeniyam slyudy [Methodical recommendations on application of reserve classification to deposits of mica]. Moscow, Ministerstvo prirodnykh resursov RF Publ., 2005. 41 p.

22. Proizvodstvo i primenenie vermikulita [Production and application of vermiculite]. Ed. by N.A. Popov. Moscow, Stroyizdat Publ., 1964. $128 \mathrm{p}$.

23. Nizhegorodov A.I. Theory and practical use of modular-pouring electric furnaces for firing vermiculite. Refractories and Industrial Ceramics, 2015, vol. 56, no. 4, pp. 361-365.

24. Nizhegorodov A.I. Testing a new alternative electric furnace for vermiculite concentrates heat treatment. Bulletin of the Tomsk Polytechnic University. Geo Assets Engineering, 2018, vol. 329, no. 4, pp. 142-153. In Rus.

Received: 28 August 2018.

\section{Information about the authors}

Anatoly I. Nizhegorodov, Dr. Sc., professor, Irkutsk National Research Technical University.

Alexey N. Gavrilin, Cand. Sc., associate professor, National Research Tomsk Polytechnic University.

Boris B. Moyzes, Cand. Sc., associate professor, National Research Tomsk Polytechnic University.

Kirill A. Kuvshinov, chief lecturer, National Research Tomsk Polytechnic University. 